\title{
Panic Disorder: Current Research and Management Approaches
}

\author{
Yong-Ku Kim, Guest Editor \\ Department of Psychiatry, College of Medicine, Korea University, Seoul, Republic of Korea
}

Panic disorder is one of the most common anxiety disorders, with lifetime prevalence rates in the general population reported between $2.1-4.7 \% .{ }^{1.2}$ Panic disorder is typically associated with a chronic progression, which results in economic burden and a loss of quality of life, therefore, proper prevention and treatment of panic disorder is important. The Diagnostic and Statistical Manual of Mental Disorders, Fifth Edition (DSM-5) was revised to accommodate a paradigm shift from a categorical to a dimensional approach in psychiatric nosology and taxonomy. However, the categorical concept of panic disorder still remains in the DSM-5. An overview of the changes to diagnostic criteria for panic disorder from the DSM-IV to DSM-5 are as follows: 1) distinction of agoraphobia from panic disorder and 2) defining panic attacks as a specifier. In the DSM-5, a panic disorder diagnosis is operationally defined as the fulfillment of both recurrent unexpected panic attacks (Criterion $\mathrm{A}$ ) and the existence of one or more of the following persistent panic attack related conditions for at least one month: concern, worry, and behavioral change (Criterion B). Panic disorder has been modeled in terms of the negative valence systems domain in the Research Domain Criteria initiative. ${ }^{3}$ A panic attack (Criteria $\mathrm{A}$ ) can be conceptualized as a prototypical expression of a fear response to an acute internal threat stimulus, while concerns and worries about the consequences of panic attacks (Criteria B) can be conceptualized as responses to potential harm within the negative valence system.

It is necessary to integrate behavioral, physiological, neurobiological, and genetic data to conceptualize a bio-psychosocial-behavioral model of panic disorder. Park and $\mathrm{Kim}^{4}$ have reviewed and discussed a novel bio-psychosocial-behavioral treatment model for panic disorder in this special issue. Candidate genes or proteins for susceptibility to panic disorder that affect different neurotransmitter systems, including serotonin, norepinephrine, adenosine, GABA and glutamate, neuropeptides, HPA axis and neurotrophins have been identified in the molecular genetics perspective of panic dis-

(c) This is an Open Access article distributed under the terms of the Creative Commons Attribution Non-Commercial License (https://creativecommons.org/licenses/bync/4.0) which permits unrestricted non-commercial use, distribution, and reproduction in any medium, provided the original work is properly cited. order. Clinically, there is a need for more effective, fast-acting and more tolerable pharmacological interventions for panic disorder, which is currently unmet. Therefore, novel mechanism-based anti-panic therapeutics, including glutamatergic receptor modulators, orexin receptor antagonists, corticotropin-releasing factor (CRF) 1 receptor antagonists, angiotensin II receptor antagonists, and endocannabinoid system modulators have been proposed as promising future drugs for panic disorders. Dysfunctional cross-talk between emotional drive (limbic structure) and cognitive inhibition (prefrontal cortex) and the fear circuit of the amygdala-hippocampal-prefrontal axis are considered to be the neuroanatomical correlates for panic disorder. The neural perspective supports the idea that cognitive-behavioral therapy normalizes alterations in the top-down cognitive process among brain structures. An essential component of "personalized medicine" is the application of reliable indicators of predictions/outcomes, which are used to select the most appropriate therapeutic for subgroups of panic disorder. Consistent with the concept of "personalized medicine," the Research Domain Criteria can provide a more precise framework for further treatments that target the underlying dysfunctions of panic disorder and provide us with better definitions of moderators that can be used to identify subgroups, according to different responses to treatment.

Several cognitive, behavioral and neurobiological theories have been proposed to explain the pathogenesis of panic disorder. Gorman et al. ${ }^{5}$ has suggested a neuroanatomic model that integrates the different views of panic disorder as either biological or a psychological disease. According to Dr. Gorman's model, fearful responses and panic attacks are mediated by a "fear network" in the brain that is centered in the amygdala and interacts with the hippocampus and medial prefrontal cortex. This neuroanatomical model has stimulated additional research, primarily in neuroimaging studies. In this special issue, $\mathrm{Lai}^{6}$ has pursued an additional theoretical approach using the traditional fear network model of panic disorder and has highlighted an extended and advanced fear network model of panic disorder. In the advanced fear network model, sensory regions of the temporal-parietal-occipital lobe have critical roles in the development of fear and anxiety. In addi- 
tion, the insular integrates filtered sensory information via the thalamus from the sensory regions of the occipital, parietal, and temporal lobes and sends the information to the frontal regions for cognitive processing, and to the limbic system for primitive response, which causes the imbalance in panic disorder. Additional extensive and advanced neuroimaging studies that focus on imaging genetics, machine learning, and pattern recognition are necessary in the future, to confirm the role of the advanced fear network model in the pathophysiology of panic disorder.

Panic disorder is a complex heterogeneous multifactorial and polygenic disorder. ${ }^{7}$ This heterogeneity not only complicates panic disorder diagnosis but also the treatment outcomes and prognosis. To date, no valid, specific, or sensitive biomarkers have been identified for panic disorder, or for the treatment response in panic disorder. In this special issue, Cosci and Mansueto ${ }^{8}$ have reviewed the biological and clinical markers of panic disorder. Until now, potential candidate biomarkers of panic disorders have been proposed, including neuroimaging, respiratory patterns, heart rate variability, peripheral blood markers, and hypothalamic-pituitary-adrenal axis dysregulation; however, the clinical utility, sensitivity, specificity, and the predictive value of the biomarkers for panic disorder remain questionable. As a solution to the problem of low sensitivity and specificity of a single biomarker, it has been suggested that a wider and multivariable approach could be applied, including a combination of neuroimaging, genetic, epigenetic, proteomic, and metabolomics approaches to include the majority of multiple biological abnormalities of panic disorder. Alternatively, a staging model for panic disorder could also be a valid approach to include the susceptibility, diagnostic, prognostic, and predictive markers of panic disorder. Biomarkers would be more specific if they could be related to specific stages of panic disorder. In this review, the authors ${ }^{8}$ shed light on the staging model of panic disorder with stagespecific biological markers.

In general, panic disorder can be effectively treated with pharmacological and psychological treatments such as cognitive behavioral therapy and mindfulness interventions. ${ }^{9} \mathrm{Al}-$ though pharmacological treatments have proven to be potent in panic disorder therapy, their potential side effects can be obstacles for adherence to treatment and the long-term maintenance of achieved treatment outcomes. Thus, it is important to provide effective psychological interventions for panic disorder either as adjunctive or stand-alone treatment. However, despite evidence-based psychological therapy programs, the availability of treatment places is limited in conventional face-to-face mental health care. In this special issue, Apolinario-Hagen ${ }^{10}$ has explored recent evidence based on the efficacy and acceptability of different internet-delivered treatments for adult patients with panic disorder. This review illustrates different effective and well accepted evidence-based internetdelivered psychological treatments for panic disorder. Of note, self-guided transdiagnostic internet cognitive behavioral therapy approaches appear to be efficient options for large scale dissemination in routine care. However, the discrepancy between high acceptability and slow uptake of internetdelivered treatments, as well as the unclear role of patients' preferences, require further investigation.

Several effective medications are available for treating panic disorder, but outcomes have been unsatisfactory in a number of patients, suggesting the usefulness of expanding the array of anti-panic drugs and improving the quality of response to current recommended treatments. ${ }^{11}$ In addition to drug therapy for panic disorder, neuromodulation techniques such as Transcranial Magnetic stimulation (TMS) have been applied in panic disorder with varying results. ${ }^{12}$ In this special issue, Zugliani et al. ${ }^{13}$ confirmed the efficacy of tranylcypromine, paroxetine, clonazepam, alprazolam, and escitalopram as effective options for treating panic disorder through a systematic review. The new antidepressant, vortioxetine and TMS, when administered for 4 or more weeks, may also be an effective treatment for panic disorder. Therefore, future studies should focus on neuromodulation techniques and new psychopharmacological compounds, and compare these new treatment approaches to the well-established and current effective treatments for panic disorder.

I sincerely thank all of the authors for their valuable time that was spent preparing manuscripts. The authors are leading scientists with knowledge and expertise in panic disorder. I hope this special issue is helpful and provides insight for better understanding and treating panic disorder.

\section{REFERENCES}

1. Baxter AJ, Vos T, Scott KM, Ferrari AJ, Whiteford HA. The global burden of anxiety disorders in 2010. Psychol Med 2014;44:2363-2374.

2. Carta MG, Moro MF, Aguglia E, Balestrieri M, Caraci F, Dell'Osso L, et al. The attributable burden of panic disorder in the impairment of quality of life in a national survey in Italy. Int J Soc Psychiatry 2015;61:693699.

3. Insel T, Cuthbert B, Garvey M, Heinssen R, Pine DS, Quinn K, et al. Research domain criteria (RDoC): toward a new classification framework for research on mental disorders. Am J Psychiatry 2010;167:748751.

4. Park SC, Kim YK. A novel bio-psychosocial-behavioral treatment model of panic disorder. Psychiatry Investig 2019;16:4-15.

5. Gorman JM, Kent JM, Sullivan GM, Coplan JD. Neuroanatomical hypothesis of panic disorder, revised. Am J Psychiatry 2000;157:493-505.

6. Lai $\mathrm{CH}$. Fear network model in panic disorder: the past and the future. Psychiatry Investig 2019;16:16-26.

7. Kim EJ, Kim HJ, Kim YK. Genetic, Epigenetic and Environmental Interaction in Panic Disorder. In: Kim YK, Editor. Panic Disorder: Assessment, Management, and Research Insights. New York: NOVA SCIENCE, 2017, p.21-39. 
8. Cosci F, Mansueto G. Biological and clinical markers in panic disorder. Psychiatry Investig 2019;16:27-36.

9. Bystritsky A, Khalsa SS, Cameron ME, Schiffman J. Current diagnosis and treatment of anxiety disorders. P T 2013;38:30-57.

10. Apolinario-Hagen J. Internet-delivered psychological treatment options for panic disorder: a review on their efficacy and acceptability. Psychiatry Investig 2019;16:37-49.

11. Caldirola D, Alciati A, Riva A, Perna G. Are there advances in pharmacotherapy for panic disorder? A systematic review of the past five years.
Expert Opin Pharmacother 2018;19:1357-1368.

12. Iannone A, Cruz AP, Brasil-Neto JP, Boechat-Barros R. Transcranial magnetic stimulation and transcranial direct current stimulation appear to be safe neuromodulatory techniques useful in the treatment of anxiety disorders and other neuropsychiatric disorders. Arq Neuropsiquiatr 2016;74:829-835.

13. Zugliani M, Cabo M, Nardi AE, Perna G, Freire RC. Pharmacological and neuromodulatory treatments for panic disorder: clinical trials from 2010 to 2018. Psychiatry Investig 2019;16:50-58. 